\title{
Some theorems on absolute Cesàro summability
}

\author{
By J. M. Hyslop.
}

(Received 30th June, 1939. Read 3rd November, 1939.)

1. It is convenient to begin with a brief statement of the notation which will be used throughout this paper.

Let $k$ be any positive number and let

$$
S_{n}^{(k)}=\sum_{\nu=0}^{n} A_{n-\nu}^{(k)} a_{\nu}
$$

where $A_{n}^{(k)}$ is the coefficient of $x^{n}$ in the formal expansion of $(1-x)^{-k-1}$, and let

$$
c_{n}^{(k)}=S_{n}^{(k)} / A_{n}^{(k)}, a_{n}^{(k)}=c_{n}^{(k)}-c_{n-1}^{(k)} .
$$

Then the series $\Sigma a_{n}$ is said to be summable $(C, k)$ if $\Sigma a_{n}^{(k)}$ is convergent, that is, if $c_{n}^{(k)}$ tends to a limit, and absolutely summable $(C, k)$, or summable $|C, k|$, if $\Sigma a_{n}^{(k)}$ is absolutely convergent.

The symbols $S_{n, \rho}^{(k)}, c_{n, \rho}^{(k)}, a_{n, \rho}^{(k)}$ denote respectively the values of $S_{n}^{(k)}, c_{n}^{(k)}$ and $a_{n}^{(k)}$ for the series $\Sigma n^{\rho} a_{n}$. Whenever $p$ and $\rho$ occur it is to be assumed that $p$ is a positive integer and that $0<\rho<1$.

2. This paper may be regarded from one point of view as a continuation of a paper ${ }^{1}$ of mine which appeared recently in these Proceedings. In that paper it was shown that the two conditions

$$
\begin{aligned}
& c_{n}^{(p)}=s+o\left(n^{-\rho}\right), \\
& \Sigma n^{\rho} a_{n} \text { is summable }(C, p),
\end{aligned}
$$

were closely connected. When $\rho=1$ they are equivalent and when $0<\rho<1$ the first is slightly more general than the second. For absolute summability we naturally expect the analogues of (1) and (2) to be

$$
\begin{aligned}
& \Sigma\left|\Delta n^{\rho}\left\{c_{n}^{(p)}-s\right\}\right|<\infty, \\
& \Sigma n^{\rho} a_{n} \text { is summable }|C, p| .
\end{aligned}
$$

1 J. M. Hyslop, Proc. Edinburgh Math. Soc. (2), 5 (1938), 182-201. 
I do not propose to discuss here whether or not the theorems of my former paper carry over without material alteration to the absolute summability case, but am concerned with another condition allied to (4) and (5), namely

$$
\Sigma n^{p}\left|a_{n}^{(p)}\right|<\infty
$$

It will be shown in fact that (5) and (6) are equivalent for $0<\rho<1$ and that modifications are necessary in the case $\rho=1$.

Dr Bosanquet, to whom I communicated this result, has pointed out that its analogue for ordinary summability, at any rate in the case $0<\rho<1$, can be deduced from a theorem of Andersen, the analogue of $(6)$ being of course

$$
\Sigma n^{\rho} a_{n}^{(p)} \text { is convergent. }
$$

3. Several lemmas will be required for the proofs of the main theorems.

LEMMA 1. If $k>0$ we have

$$
S_{n, 1}^{(k)}=(k+n+1) S_{n}^{(k)}-(k+1) S_{n}^{(k+1)} .
$$

This result is well known and easy to prove.

Lemma 2. We have formally, for $k>0$,

$$
\sum_{n=1}^{\infty} n A_{n}^{(k)} a_{n}^{(k)} x^{n}=(1-x)^{-k} \sum_{n=0}^{\infty} n a_{n} x^{n} .
$$

LEMMA 3. If $r$ is any real number and $p$ is a positive integer ${ }^{3}$

where

$$
\sum_{\nu=\mu}^{n} A_{n-\nu}^{(r)} A_{\nu-\mu}^{(-p-1)} \epsilon_{r}=\sum_{q=0}^{p}\left(\begin{array}{c}
p \\
q
\end{array}\right) A_{n-\mu-q}^{(r+q-p)} \Delta^{q} \epsilon_{\mu},
$$

$$
\Delta^{q} \epsilon_{\mu}=\epsilon_{\mu}-\left(\begin{array}{c}
q \\
1
\end{array}\right) \epsilon_{\mu+1}+\left(\begin{array}{l}
q \\
2
\end{array}\right) \epsilon_{\mu+2} \ldots+(-1)^{q} \epsilon_{\mu+q} .
$$

LemMa 4. If $0 \leqq \sigma<k+1$ and if $\Sigma n^{\sigma}\left|a_{n}^{(k)}\right|$ is convergent then so also is $\Sigma n^{\sigma}\left|a_{n}^{(k+\delta)}\right|$, for every $\delta>0$.

This lemma reduces simply to the theorem of consistency for absolute Cesàro summability in the case when $\sigma=0$.

${ }^{1}$ A. F. Andersen, Proc. London Math. Soc. (2), 27 (1928), 39-71.

2 E. Kogbetlianbz, Bull. des Sciences Math. (2), 49 (1925), 234-256.

${ }^{3}$ See A. F. Andersen, Studier over Cesàro's Summatilitetsmetode (Copenhagen, 1921), 42, and J. M. Hyslop, loc. cit., 187. 
We have, from Lemma 2,

$$
n A_{n}^{(k+\delta)} a_{n}^{(k+\delta)}=\sum_{\nu=1}^{n} A_{n-\nu}^{(\delta-1)} \nu A_{\nu}^{(k)} a_{\nu}^{(k)}
$$

whence ${ }^{1}$, taking $\delta<1$, as we obviously may,

$$
\begin{aligned}
\sum_{n=1}^{\infty} n^{\sigma}\left|a_{n}^{(k+\delta)}\right| & <A \sum_{n=1}^{\infty}(n+1)^{\sigma-k-\delta-1}\left|\sum_{\nu=1}^{n} A_{n-\nu}^{(\delta-1)} \nu A_{\nu}^{(k)} a_{\nu}^{(k)}\right| \\
& <A \sum_{\nu=1}^{\infty} \nu^{k+1}\left|a_{\nu}^{(k)}\right| \sum_{n=\nu}^{\infty}(n+1)^{\sigma-k-\delta-1}(n-\nu+1)^{\delta-1} \\
& <A \sum_{\nu=1}^{\infty} \nu^{k+1}\left|a_{\nu}^{(k)}\right| \int_{\nu}^{\infty} x^{\sigma-k-\delta-1}(x-\nu)^{\delta-1} d x \\
& <A \sum_{\nu=1}^{\infty} \nu^{\sigma}\left|a_{\nu}^{(k)}\right| \int_{1}^{\infty} y^{\sigma-k-\delta-1}(y-1)^{\delta-1} d y \\
& <A
\end{aligned}
$$

4. We proceed now to obtain the equivalence theorem.

Theorem 1. If the series $\Sigma n^{\rho}\left|a_{n}^{(p)}\right|$ is convergent then the series $\sum n^{\rho} a_{n}$ is summable $|C, p|$.

From Lemma 2 we have

where

$$
\begin{aligned}
n A_{n}^{(p)} a_{n, \rho}^{(p)} & =\sum_{\nu=1}^{n} A_{n-\nu}^{(p-1)} \nu^{\rho+1} a_{\nu} \\
& =\sum_{\nu=1}^{n} A_{n-\nu}^{(p-1)} \nu^{\rho} \sum_{\mu=1}^{\nu} A_{\nu-\mu}^{(-p-1)} \mu A_{\mu}^{(p)} a_{\mu}^{(p)} \\
& =\sum_{\mu=1}^{n} \mu A_{\mu}^{(p)} a_{\mu}^{(p)} \sum_{\nu=\mu}^{n} \nu^{\rho} A_{n-\nu}^{(p-1)} A_{\nu-\mu}^{(-p-1)} \\
& =E_{1}+E_{2},
\end{aligned}
$$

$$
\begin{aligned}
& E_{1}=\sum_{\mu=n-p+1}^{n} \mu A_{\mu}^{(p)} a_{\mu}^{(p)} \sum_{\nu=\mu}^{n} \nu^{\rho} A_{n-\nu}^{(p-1)} A_{\nu-\mu}^{(-p-1)}, \\
& E_{2}=\sum_{\mu=1}^{n-p} \mu A_{\mu}^{(p)} a_{\mu}^{(p)} \sum_{\nu=\mu}^{n} \nu^{\rho} A_{n-\nu}^{(p-1)} A_{\nu-\mu}^{(-p-1)}
\end{aligned}
$$

Clearly $\Sigma n^{-p-1} \mid E_{1}$ ! is convergent and we have to prove that the same is true of $E_{2}$.

1 Here as elsewhere $A$ is independent of the variables under consideration and has not necessarily the same value each time it occurs. 


\section{By Lemma 3 we may write}

where

$$
\begin{aligned}
E_{2} & =\sum_{\mu=1}^{n-p} \mu .4_{\mu}^{(p)} a_{\mu}^{(p)} \sum_{q=0}^{p}\left(\begin{array}{l}
p \\
q
\end{array}\right) A_{n-\mu-q}^{(q-1)} \Delta^{q} \mu^{\rho} \\
& =\sum_{q=0}^{p}\left(\begin{array}{l}
p \\
q
\end{array}\right) F_{q},
\end{aligned}
$$

$$
F_{q}=\sum_{\mu=1}^{n-p} \mu A_{\mu}^{(p)} a_{\mu}^{(p)} A_{n-\mu-q}^{(q-1)} \Delta^{q} \mu^{\rho}
$$

Now $F_{0}=0$ since $A_{n-\mu}^{(-1)}=0$. Using the inequality

$$
\Delta^{q} \mu^{\rho}<A \mu^{\rho-q}
$$

we obtain, for $q=1,2, \ldots, p$,

$$
\begin{aligned}
\sum_{n=p+1}^{\infty} n^{-p-1}\left|F_{q}\right| & <A \sum_{n=p+1}^{\infty} n^{-p-1} \sum_{\mu=1}^{n-p} \mu^{p+1+\rho-q}(n-\mu-q+1)^{q-1}\left|a_{\mu}^{(p)}\right| \\
& <A \sum_{\mu=1}^{\infty} \mu^{p+1+\rho-q}\left|a_{\mu}^{(p)}\right| \sum_{n=\mu+p}^{\infty} n^{-p-1}(n-\mu-q+1)^{q-1} \\
& <A \sum_{\mu=1}^{\infty} \mu^{p+1+\rho-q} ; a_{\mu}^{(p)} \mid \sum_{n=\mu+p}^{\infty} n^{-p+q-2} \\
& <A \sum_{\mu=1}^{\infty} \mu^{\rho}\left|a_{\mu}^{(p)}\right| \\
& <A .
\end{aligned}
$$

The theorem is therefore proved and we turn now to the converse.

Theorem 2. If the series $\sum n^{\rho} a_{n}$ is summable $|C, p|$ then the series $\Sigma n^{\rho}\left|a_{n}^{(p)}\right|$ is convergent.

As in the proof of the previous theorem we may write

where

$$
n A_{n}^{(p)} a_{n}^{(p)}=E_{1}+E_{2}
$$

$$
\begin{aligned}
& E_{1}=\sum_{\mu=n-p+1}^{n} \mu A_{\mu}^{(p)} a_{\mu, \rho}^{(p)} \sum_{\nu=\mu}^{n} A_{n-\nu}^{(p-1)} A_{\nu-\mu}^{(-p-1)} \nu-\rho \\
& E_{2}=\sum_{\mu=1}^{n-p} \mu A_{\mu}^{(p)} a_{\mu, \rho}^{(p)} \sum_{\nu=\mu}^{n} A_{n-\nu}^{(p-1)} A_{\nu-\mu}^{(-p-1)} \nu^{-\rho} .
\end{aligned}
$$

Clearly $\Sigma n^{p-p-1}\left|E_{\jmath}\right|$ is convergent and we must prove that this is also true of $E_{2}$.

By Lemma 3 we may write

$$
E_{2}=\sum_{q=0}^{p}\left(\begin{array}{l}
p \\
q
\end{array}\right) F_{q},
$$


where

$$
F_{q}=\sum_{\mu=1}^{n-p} \mu A_{\mu}^{(p)} a_{\mu, \rho}^{(p)} A_{n-\mu-q}^{(q-1)} \Delta^{q} \mu^{-p} .
$$

Now $F_{0}=0$ and, for $q=1,2, \ldots, p$,

$$
\begin{aligned}
\sum_{n=p+1}^{\infty} n^{\rho-p-1}\left|F_{q}\right| & <A \sum_{\mu=1}^{\infty} \mu^{p+1-\rho-q} \mid a_{\mu, \rho}^{(p)} \sum_{n=\mu+p}^{\infty} n^{\rho-p-1}(n-\mu-q+1)^{q-1} \\
& <A \sum_{\mu=1}^{\infty} \mu^{p+1-\rho-q}\left|a_{\mu, \rho}^{(p)}\right| \sum_{n=\mu+p}^{\infty} n^{\rho+q-p-2} \\
& <A \sum_{\mu=1}^{\infty}\left|a_{\mu, \rho}^{(p)}\right| \\
& <A .
\end{aligned}
$$

The theorem is therefore proved and the equivalence of (5) and (6) is established.

5. We now consider the case $\rho=1$ of the preceding theorems.

Theorem 3. If the series $\Sigma n\left|a_{n}^{(p)}\right|$ is convergent then the series $\Sigma n a_{n}$ is summable $|C, p|$.

This may be obtained simply by putting $\rho=1$ in the proof of Theorem 1. The theorem, however, is true not merely when $p$ is a positive integer but also when $p$ is any positive number, as the following simple argument shows.

We have

$$
\begin{aligned}
n A_{n}^{(p)} a_{n, 1}^{(p)} & =\sum_{\mu=1}^{n} \mu A_{\mu}^{(p)} a_{\mu}^{(p)} \sum_{\nu=\mu}^{n} \nu A_{n-\nu}^{(p-1)} A_{\nu-\mu}^{(-p-1)} \\
& =\sum_{\mu=1}^{n} \mu A_{\mu}^{(p)} a_{\mu}^{(p)} \sum_{\nu=\mu}^{n}\{n-(n-\nu)\} A_{n-\nu}^{(p-1)} A_{\nu-\mu}^{(-p-1)} \\
& =n \sum_{\mu=1}^{n} \mu A_{\mu}^{(p)} a_{\mu}^{(p)} A_{n-\mu}^{(-1)}-p \sum_{\mu=1}^{n-1} \mu A_{\mu}^{(p)} a_{\mu}^{(p)} \sum_{\nu=\mu}^{n-1} A_{n-1-\nu}^{(p)} A_{\nu-\mu}^{(-p-1)} \\
& =n^{2} A_{n}^{(p)} a_{n}^{(p)}-p \sum_{\mu=1}^{n-1} \mu A_{\mu}^{(p)} a_{\mu}^{(p)} A_{n-1-\mu}^{(0)} \\
& =n^{2} A_{n}^{(p)} a_{n}^{(p)}-p \sum_{\mu=1}^{n-1} \mu A_{\mu}^{(p)} a_{\mu}^{(p)}
\end{aligned}
$$

Hence

$$
\begin{aligned}
\sum_{n=2}^{\infty}\left|a_{n, 1}^{(p)}\right| & <A \sum_{n=2}^{\infty} n\left|a_{n}^{(p)}\right|+A \sum_{\mu=2}^{\infty} \mu A_{\mu}^{(p)}\left|a_{\mu}^{(p)}\right| \sum_{n=\mu}^{\infty} n^{-p-1} \\
& <A
\end{aligned}
$$


Theorem 4. If the series $\Sigma n a_{n}$ is summable $|C, p|$ then

\section{is convergent.}

$$
\sum_{n=1}^{\infty}\left|n a_{n}^{(p)}-\frac{p}{n} c_{n, 1}^{(p)}\right|
$$

As in the proof of Theorem 2 we have

where

$$
\begin{aligned}
n A_{n}^{(p)} a_{n}^{(p)}= & \sum_{\mu=}^{n} \mu A_{\mu}^{(p)} a_{\mu, 1}^{(p)} \sum_{\nu=\mu}^{n} A_{n-\nu}^{(p-1)} A_{\nu-\mu}^{(-p-1)} \nu^{-1} \\
& +\sum_{q=0}^{p-1}\left(\begin{array}{c}
p \\
q
\end{array}\right) F_{q}+\sum_{\mu=1}^{n-p} \mu A_{\mu}^{(p)} a_{\mu, 1}^{(p)} A_{n-\mu-p}^{(p-1)} \Delta^{p} \mu^{-1},
\end{aligned}
$$

Now

$$
F_{q}=\sum_{\mu=1}^{n-p} \mu A_{\mu}^{(p)} a_{\mu, 1}^{(p)} A_{n-\mu-q}^{(q-1)} \Delta^{q} \mu^{-1}
$$

$$
\sum_{n=p+1}^{\infty} \frac{1}{A_{n}^{(p)}}{ }_{\mu=n-p+1}^{n} \mu A_{\mu}^{(p)} a_{\mu, 1}^{(p)} \sum_{\nu=\mu}^{n} A_{n-\nu}^{(p-1)} A_{\nu-\mu}^{(-p-1)} \nu^{-1}\left|<A \sum_{\mu=1}^{\infty}\right| a_{\mu, 1}^{(p)} \mid
$$

Also $F_{0}=0$ and, for $q=1,2, \ldots, p-1$,

$$
<A \text {. }
$$

$$
\begin{aligned}
\sum_{n=p+1}^{\infty} \frac{1}{A_{n}^{(p)}}\left|\bar{F}_{q}\right| & <A \sum_{\mu=1}^{\infty} \mu^{p-q}\left|a_{\mu, 1}^{(p)}\right| \sum_{n=\mu+p}^{\infty} n^{-p}(n-\mu-q+1)^{q-1} \\
& <A \sum_{\mu=1}^{\infty} \mu^{p-q}\left|a_{\mu, 1}^{(p)}\right| \sum_{n=\mu+p}^{\infty} n^{q-p-1} \\
& <A \sum_{\mu=1}^{\infty}\left|a_{\mu, 1}^{(p)}\right| \\
& <A .
\end{aligned}
$$

It follows that

and, since

$$
\sum_{n=p+1}^{\infty}\left|n a_{n}^{(p)}-\frac{1}{A_{n}^{(p)}} \sum_{\mu=1}^{n-p} A_{n-\mu-p}^{(p-1)} a_{\mu, 1}^{(p)} \mu A_{\mu}^{(p)} \Delta_{!}^{p_{!}^{\natural} \mu^{-1}}\right|<\infty,
$$

we obtain

$$
\mu A_{\mu}^{(p)} \Delta^{p} \mu^{-1}=1
$$

Now

$$
\sum_{n=p}^{\infty} \mid n a_{n}^{(p)}-\frac{1}{A_{n}^{(p)}} \sum_{\mu=1}^{n-p} A_{n-p-\mu}^{(p-1)} a_{\mu, 1}^{(p)}<\infty \text {. }
$$

$$
\begin{aligned}
\sum_{n=p+1}^{\infty} \frac{1}{A_{n}^{(p)}} \mid & \sum_{\mu=1}^{n-p}\left\{A_{n-p}^{(p-1)}-A_{n-p-\mu}^{(p-1)}\right\} a_{\mu, 1}^{(p)} \mid \\
& <A \sum_{n=p}^{\infty} n-p \sum_{\mu=1}^{n-p}(n-p+1)^{p-2} \mu\left|a_{\mu, 1}^{(p)}\right| \\
& <A \sum_{\mu=1}^{\infty} \mu\left|a_{\mu, 1}^{(p)}\right| \sum_{n=\mu+p}^{\infty} n^{-p}(n-p+1)^{p-2} \\
& <A \sum_{\mu=1}^{\infty}\left|a_{\mu, 1}^{(p)}\right|<A,
\end{aligned}
$$


and it therefore follows that

that is,

$$
\sum_{n=p}^{\infty} \mid n a_{n}^{(p)}-\frac{A_{n-p}^{(p-1)}}{A_{n}^{(p)}} \sum_{\mu=1}^{n-p} a_{\mu, 1}^{(p)}<\infty
$$

$$
\sum_{n=p}^{\infty} \vdots n a_{n}^{(p)}-\left\{\frac{p}{n}+O\left(\frac{1}{n^{2}}\right)\right\}\left\{c_{n, 1}^{(p)}-\sum_{\mu=n-p+1}^{n} a_{\mu, 1}^{(p)}\right\}<\infty .
$$

Since $c_{n, 1}^{(p)}$ tends to a finite limit as $n \rightarrow \infty$ it is clear that the series

$$
\sum_{n=p}^{\infty} n^{-2}\left|c_{n, 1}^{(p)}\right|, \sum_{n=p}^{\infty} n^{-2} \mid \sum_{\mu=n-p+1}^{n} a_{\mu, 1}^{(p)}
$$

are convergent. Moreover

$$
\sum_{n=p}^{\infty} n^{-1} \sum_{\mu=n-p+1}^{n}\left|a_{\mu, 1}^{(p)}\right|<\sum_{\mu=1}^{\infty}\left|a_{\mu, 1}^{(p)}\right| \sum_{n=\mu}^{\mu+p} n^{-1}<A .
$$

The theorem is therefore proved.

The theorem shows that, when the series $\Sigma n a_{n}$ is summable $|C, p|$ to a non-zero sum, the series $\Sigma n\left|a_{n}^{(p)}\right|$ is always divergent. When the sum of the series $\Sigma n a_{n}$ is zero the convergence or divergence of $\Sigma n\left|a_{n}^{(p)}\right|$ depends on the convergence or divergence of the series $\Sigma n^{-1}\left|c_{n_{1} 1}^{(p)}\right|$. That either alternative may occur is easily seen by considering the example

For this series

$$
\begin{aligned}
& a_{0}=a_{1}=0, \\
& a_{n}=n^{-1} \sum_{\nu=2}^{n} A_{n-\nu}^{(-p-2)} A_{\nu}^{(p)}(\log \nu)^{-a},(n \geqq 2) .
\end{aligned}
$$

When $\alpha=1$,

$$
c_{n, 1}^{(p)}=(\log n)^{-a},(n \geqq 2) .
$$

$$
a_{n, 1}^{(p)}=O\left\{n^{-1}(\log n)^{-2}\right\}
$$

so that the series $\Sigma n a_{n}$ is summable $|C, p|$ to the sum zero and $\sum n^{-1}\left|c_{n, 1}^{(p)}\right|$ is divergent. When $a=2$,

$$
a_{n, 1}^{(p)}=O\left\{n^{-1}(\log n)^{-3}\right\}
$$

so that the series $\Sigma n a_{n}$ is again summable $|C, p|$ to the sum zero but $\Sigma n^{-1}\left|c_{n, 1}^{(p)}\right|$ is convergent.

6. We conclude by proving a theorem which is rather more precise than Theorem, 4. For the proof we require two lemmas. 
Lемma 5. If the series $\Sigma n a_{n}$ is summable $|C, p|$ then

$$
\sum_{n=1}^{\infty}\left|n a_{n}^{(p+\lambda)}-\frac{p+\lambda}{n} c_{n, 1}^{(p+\lambda)}\right|<\infty,
$$

where $\lambda$ is zero or any positive integer.

This is an immediate corollary from Theorem 4 and the consistency theorem for absolute Cesàro summability.

LEMMA 6. If $k>0$ we have

$$
\frac{a_{n, 1}^{(k)}}{k+n}-\frac{c_{n, 1}^{(k)}}{(k+n)(k+n+1)}=a_{n}^{(k)}-a_{n}^{(k+1)} .
$$

From Lemma 1 we have

whence

$$
\frac{S_{n, 1}^{(k)}}{A_{n}^{(k+1)}}-\frac{S_{n-1,1}^{(k)}}{A_{n-1}^{(k+1)}}=(k+1)\left\{a_{n}^{(k)}-a_{n}^{(k+1)}\right\}
$$

$$
\begin{aligned}
a_{n}^{(k)}-a_{n}^{(k+1)} & =\frac{c_{n, 1}^{(k)}}{k+n+1}-\frac{c_{n-1,1}^{(k)}}{k+n} \\
& =\frac{a_{n, 1}^{(k)}}{k+n}+c_{n, 1}^{(k)}\left\{\frac{1}{k+n+1}-\frac{1}{k+n}\right\}
\end{aligned}
$$

which yields the required result.

Theorem 5. Suppose that the series $\Sigma n a_{n}$ is summable $|C, p|$. If the series $\Sigma n^{-1}\left|c_{n, 1}^{(p)}\right|$ is convergent then $\Sigma n\left|a_{n}^{(k)}\right|$ is convergent for $k=p$. If the series $\Sigma n^{-1}\left|c_{n, 1}^{(p)}\right|$ is divergent then $\Sigma n\left|a_{n}^{(k)}\right|$ is divergent for every value of $k$.

The first part of the theorem has already been proved. We proceed to prove the second part.

Suppose that $\Sigma n^{-1}\left|c_{n, 1}^{(p)}\right|=\infty$. Then, from Lemma 5, with $\lambda=0$, we have $\Sigma n\left|a_{n}^{(p)}\right|==\infty$. From Lemma 6, we may write

$$
n a_{n}^{(p+1)}=\left\{n a_{n}^{(p)}-\frac{p}{n} c_{n, 1}^{(p)}\right\}+\left\{\frac{p}{n}+\frac{n}{(p+n)(p+n+1)}\right\} c_{n, 1}^{(p)}-\frac{n a_{n, 1}^{(p)}}{p+n}
$$

Now

while

$$
\Sigma^{\prime} n a_{n}^{(p)}-\frac{p}{n} c_{n, 1}^{(p)}\left|<\infty, \Sigma \frac{n}{p+n}\right| a_{n .1}^{(p)} \mid<\infty
$$

It follows that

$$
\Sigma\left\{\frac{p}{n}+\frac{n}{(p+n)(p+n+1)}\right\}\left|c_{n, 1}^{(p)}\right|=\infty .
$$

$$
\sum n\left|a_{n}^{(p+1)}\right|=\infty,
$$

and from Lemma 5, with $\lambda=1$, we obtain

$$
\Sigma n^{-1}\left|c_{n, 1}^{(p+1)}\right|=\infty \text {. }
$$


Repetition of the above argument then yields

$$
\sum n\left|a_{n}^{(p+2)}\right|=\infty \text {, }
$$

and so on.

The theorem is therefore proved for any integral value of $k \geqq p$ and the proof for general $k>0$ follows at once from this and Lemma 4 .

7. It will be observed that in this paper no attempt has been made to deal exhaustively with all the relations connecting conditions (4), (5) and (6). Indeed (4) has merely been mentioned since it seems to be the natural analogue of (I). I hope to discuss condition (4) in a later communication and also to obtain a complete set of relations between the conditions in each of the two groups. It has already been shown' that the groups themselves are to some extent inter-related.

J. M. Hyslop, Pฯoc. Edinburgh Math. Soc. (2), 6 (1939), $51-56$.

The University,

Glasgow. 